\title{
Older Familial Hypercholesterolemia Patients with COVID-19
}

\author{
Alpo Vuorio $^{\text {a, b }}$ Petri T. Kovanen ${ }^{c}$ Timo E. Strandberg ${ }^{\text {d, e }}$ \\ ${ }^{a}$ Mehiläinen, Airport Health Center, Vantaa, Finland; ${ }^{b}$ Department of Forensic Medicine, University of \\ Helsinki, Helsinki, Finland; ' Wihuri Research Institute, Helsinki, Finland; dUniversity of Helsinki and \\ Helsinki University Hospital, Helsinki, Finland; ${ }^{e}$ Center for Life Course Health Research, University of Oulu, \\ Oulu, Finland
}

\section{Keywords}

Old · COVID-19 · Statins · PCSK9 inhibitors · Atherosclerosis

The heterozygous form of familial hypercholesterolemia (FH) affects about one out of 200-250 individuals or over 30 million individuals worldwide. The risk for an atherosclerotic cardiovascular event in the $\mathrm{FH}$ population is very high, as illustrated by the fact that, on average, only one in 5 untreated $\mathrm{FH}$ men reach 70 years of age. The poor prognosis of $\mathrm{FH}$ patients has improved with the advent of statins in the late 1980s, current life expectancy of the statin-treated FH patients being similar to that of the general population [1]. Unfortunately, however, the awareness of coronary heart disease risk factors is still insufficient among older adults [2], and, moreover, despite LDL-cholesterol lowering, older patients with $\mathrm{FH}$ are still at increased risk for cardiac events $[3,4]$.

In general, the risk of patients with $\mathrm{FH}$ is well illustrated by their cumulative cholesterol burden (LDL-cholesterol [LDL-C] $\mathrm{mmol} / \mathrm{L} \times$ years) [5]. Using this metric, we can estimate that the cholesterol burden of a 70-yearold patient with $\mathrm{FH}$ and with an LDL-C level of $5 \mathrm{mmol} / \mathrm{L}$ is 350. Similarly, a patient with FH who has started statin

karger@karger.com

(c) 2021 S. Karger AG, Basel

www.karger.com/ger

Karger! therapy at the age of 30 and has reached a constant LDL$\mathrm{C}$ level of $2.5 \mathrm{mmol} / \mathrm{L}$, would have a cholesterol burden of 250 at the age of 70 . The significant decrease in the burden would then translate into an improved cardiovascular prognosis.

Of note, a typical statin-treated adult patient with $\mathrm{FH}$ has a higher cholesterol burden than a normocholesterolemic person of the same age and with a similar LDL-C level. This is because in FH patients the level of LDL-C has been significantly elevated since birth, while the diagnosis of $\mathrm{FH}$ and its treatment typically occurs only in middle age. Because of the high LDL-cholesterol burden, FH patients have subclinical atherosclerosis already in their thirties. Therefore, current lipid guidelines commonly advise $<1.8 \mathrm{mmol} / \mathrm{L}$ as the target level of LDL-C for an adult $\mathrm{FH}$ patient [3]. Often, this level can be reached only by adding a proprotein convertase subtilisin/kexin-type 9 (PCSK9) inhibitor to the usual treatment regimen, that is, to a maximally tolerated dose of statin plus ezetimibe [3].

Also, patients with $\mathrm{FH}$ tend to have elevated levels of serum lipoprotein(a) (Lp[a]) further increasing their risk of atherothrombotic events [6]. Accordingly, an elevated serum LDL-C level and an elevated Lp(a) level jointly promote endothelial dysfunction, which, together with 
the viral attack on the endothelial cells, jointly contribute to the often lethal micro- or macrothrombotic events in severe COVID-19 [7]. Regarding the myocardial damage, a recent meta-analysis revealed high numbers of acute myocardial infarction in hospitalized patients with $\mathrm{CO}$ VID-19, the prevalence being 3.3\% (95\% CI, 0.3-8.5) [8].

There is accumulating evidence that ongoing statin treatment is associated with lower inhospital mortality risk among COVID-19 patients $(N=8,990)$ [9]. In a recent meta-analysis including COVID-19 patients aged 58-75 years, the risk of death or severe disease was significantly lower with statin treatment $(\mathrm{HR}=0.70$; 95\% CI 0.53-0.94). Although the studies included in this metaanalysis were retrospective and observational, the results in the different individual studies were quite consistent supporting the benefit of ongoing statin therapy in COVID-19 patients.

In assessing the risk of an older patient with $\mathrm{FH}$, we should also consider the age-related increase in cardiac events in patients with COVID-19. Retrospective comparison of 60-74-year old and $\geq 75$-year old patients with COVID-19 revealed that during hospitalization acute cardiac injury was present in $1.2 \%$ and $20 \%$ in the younger and older age-group, respectively $(p=0.002)$ [10].

Consequently, older patients with $\mathrm{FH}$ can be considered to be at specifically high risk of cardiac events during COVID-19. Therefore, intensifying LDL-C lowering seems vital, and, moreover, the ongoing lipid-lowering therapy should be continued during the COVID-19 [11]. The clinical benefit of a PCSK9 inhibitor was recently demonstrated in a patient population with both an elevated LDL-C and Lp(a) level, and the reduction of both lipids contributed independently to cardiovascular event reduction [12]. Therefore, patients with $\mathrm{FH}$, having a life-long double risk burden, might particularly gain when a PCSK 9 inhibitor is added to the conventional lipid treatment. In addition, early intervention with a PCSK9 inhibitor as an adjunct therapy has been suggested for patients with COVID-19 $[11,13]$. The potential benefits of the PCSK9 in- hibitors may include augmentation of endothelial dysfunction and improvement of cellular antiviral defense [14]. However, the proposed clinical benefits of the intensification of lipid-lowering therapies with PCSK9 inhibitors should be tested in randomized and controlled trials in hospitalized COVID-19 patients with and without FH.

We have earlier introduced the concept of a "rescue" therapy with PCSK9 inhibitors for middle-aged patients having their $\mathrm{FH}$ diagnosis at the age of 40 years or older [15]. Albeit COVID-19 likely poses also a longer term atherothrombotic threat for the high-risk patients with $\mathrm{FH}$, for cost reasons this treatment could only be temporary. However, we can conclude that an intensified treatment of hypercholesterolemia in older patients with $\mathrm{FH}$ having COVID-19 needs special attention. Thus, in addition to antecedent statin administration, at least a temporary intensification of LDL-C lowering with a PCSK9 inhibitor should be considered when the diagnosis of the viral disease is made in a patient belonging to this highrisk older patient population.

\section{Conflict of Interest Statement}

Dr. Vuorio reports no conflicts of interest. Prof. Kovanen has received consultancy fees, lecture honoraria, and/or travel fees from Amgen, Novartis, Raisio Group, and Sanofi. Prof. Strandberg has had educational, consultative, and research collaboration with several companies (incl. Amgen, Merck, OrionPharma, Sankyo, Servier, and Sanofi) marketing cholesterol-lowering drugs.

\section{Funding Sources}

The authors did not receive any funding.

\section{Author Contributions}

All authors listed have made a substantial, direct, and intellectual contribution to the work and approved it for publication.

\section{References}

FH and COVID-19
1 Humphries SE, Cooper JA, Seed M, Capps N, Durrington PN, Jones B, et al. Coronary heart disease mortality in treated familial hypercholesterolaemia: update of the UK Simon Broome FH register. Atherosclerosis. 2018; 274:41-6.

2 Mazalin Protulipac J, Sonicki Z, Reiner Ž. Cardiovascular disease (CVD) risk factors in older adults: perception and reality. Arch Gerontol Geriatr. 2015;61:88-92.
3 Mach F, Baigent C, Catapano AL, Koskinas KC, Casula M, Badimon L, et al. 2019 ESC/ EAS guidelines for the management of dyslipidaemias: lipid modification to reduce cardiovascular risk. Eur Heart J. 2020;41(1):11188.

4 Coutinho ER, Miname MH, Rocha VZ, Bittencourt MS, Jannes CE, Tada MT, et al. Familial hypercholesterolemia and cardiovascular disease in older individuals. Atheroclerosis. $2021 \mathrm{Feb} ; 318: 32-7$. 
5 Vuorio A, Docherty KF, Humphries SE, Kuoppala J, Kovanen PT. Statin treatment of children with familial hypercholesterolemia: trying to balance incomplete evidence of long-term safety and clinical accountability: are we approaching a consensus? Atherosclerosis. 2013;226:315-20.

6 Vuorio A, Watts GF, Schneider WJ, Tsimikas S, Kovanen PT. Familial hypercholesterolemia and elevated lipoprotein(a): double heritable risk and new therapeutic opportunities. J Intern Med. 2019;287(1):2-18.

7 Vuorio A, Kovanen PT. Prevention of endothelial dysfunction and thrombotic events in COVID-19 patients with familial hypercholesterolemia. J Clin Lipidol. 2020;14(5):6178.

8 Kunutsor SK, Laukkanen JA. Incidence of venous and arterial thromboembolic complica- tions in COVID-19: a systematic review and meta-analysis. Thromb Res. 2020;196:27-30.

9 Kow CS, Hasan SS. Meta-analysis of effect of statins in patients with COVID-19. Am J Cardiol. 2020;134:153-5.

10 Guo T, Shen Q, Guo W, He W, Li J, Zhang Y, et al. Clinical characteristics of elderly patients with COVID-19 in Hunan Province, China: a multicenter, prospective study. Gerontology. 2020;66:467-75.

11 Iqbal Z, Ho JH, Adam S, France M, Syed A, Neely D, et al. Managing hyperlipidaemia in patients with COVID-19 and during its pandemic: an expert panel position statement from HEART UK. Atherosclerosis. 2020;313: 126-36.

12 Szarek M, Bittner VA, Aylward P, BaccaraDinet M, BhattDL, DiazR, etal.Lipoprotein(a) lowering by alirocumab reduces the total bur- den of cardiovascular events independent of low-density lipoprotein cholesterol lowering: ODYSSEY OUTCOMES trial. Eur Heart J. 2020 Nov 21;41(44):4245-55.

13 Vuorio A, Watts GF, Kovanen PT. Familial hypercholesterolaemia and COVID-19: triggering of increased sustained cardiovascular risk. J Intern Med. 2020;287(6):746-7.

14 Vuorio A, Kovanen PT. PCSK9 inhibitors for COVID-19: an opportunity to enhance the antiviral action of interferon in patients with hypercholesterolemia. J Intern Med. 2020 Nov 21.

15 Vuorio A, Watts GF, Kovanen PT. Rescue therapy with PCSK9 inhibitors for patients with delayed diagnosis of heterozygous familial hypercholesterolemia: redressing the balance of missed opportunities. J Clin Lipidol. 2016;10(5):1278-9. 